\title{
THE NATURE AND POSTNATAL DEVELOPMENT OF REFLEX DEGLUTITION IN THE KITTEN
}

\author{
Tadaaki SumI \\ Department of Physiology, Shinshu University Medical School, \\ Matsumoto, Japan
}

The act of swallowing requires the coordination of structures in the medulla oblongata with those in the oropharyngeal region through interconnecting nervous pathways ${ }^{1,3}$. It is not known, however, when this reflex becomes fully operative.

The present study aimed at determining 1) the nature of the motor activity of the hypoglossal and phrenic nerves during reflex swallowing in the kitten, 2) the postnatal development of the integrative action of the nervous system concerning this reflex and 3) the relation of nervous actions subserving respiration and swallowing.

\section{METHODS}

Eight adult cats (over one year from the birth) and 43 kittens of various postnatal periods (ranged from 24 hours to 5 months) were used. Decerebration at the midcollicular level was done by suction with the aid of a fine glass tube under temporary ether anesthesia and the animals were tracheotomized to insure adequate ventilation. Isolation of the hypoglossal, accessory or phrenic nerve fibers was done by splitting the central stump of the nerve with a pair of forceps under microscopic control. The end of the nerve fiber thus dissected was placed on a fine platinum wire electrode and the activity of individual nerve fibers was fed into an R-C coupled amplifier, displayed on a dual beam oscilloscope and, when required, recorded on film. The second lead was placed tightly around the wound of the neck and grounded. Mineral oil was used to keep the exposed nervous tissue warm and moist. Swallowing was induced 1) by injecting a small amount of tap water into the oropharyngeal cavity or 2) by delivering repetitive electrical pulses of $0.1 \mathrm{msec}$ duration, 30 pulses per second and up to 10 volts to the central stump of the superior laryngeal nerve. In all cases, the occurrence of swallowing was monitored by means of a concentric needle electrode thrusted into the contralateral geniohyoid muscle. The activity of the muscle was displayed on the second beam of the oscilloscope. Artificial respiration was employed whenever spontaneous respiratory movements were arrested by intravenous injections of gallamine triethiodide (Flaxedil : $3 \mathrm{mg} / \mathrm{kg}$ ). In some cases, partial asphyxia was induced by temporary occlusion of the tracheal cannula.

Received for publication July 24, 1966.

角 忠明. 


\section{RESULTS}

In kittens, prominent activation associated with swallowing was induced in a variety of different motor nerve fibers including some, such as the accessory and the phrenic nerve fibers, having no direct participation in swallowing. In the present study, analysis of the motor activity during swallowing was carried out mainly on individual fibers of the hypoglossal and phrenic nerves.

1. The patterns of the impulse discharge in the hypoglossal nerve fibers during swallowing. The response pattern of the hypoglossal nerve fibers in kittens varied between fibers and even in the same fiber at different times. The three records at the left in FIG. 1 illustrate the responses of different fibers of the hypoglossal nerve induced by squirting a small amount of water when the animal was breathing quietly. If these were taken as a control the responses were enhanced by squirting a large amount of water into the oropharyngeal cavity (FIG. 1B), mechanical pressure on the oropharyngeal mucosa (FIG. 1C) or a small squirt of the water into the oropharyngeal cavity during light asphyxia (FIG. 1A). On the other hand, the discharge pattern of the

\section{CONTROL}
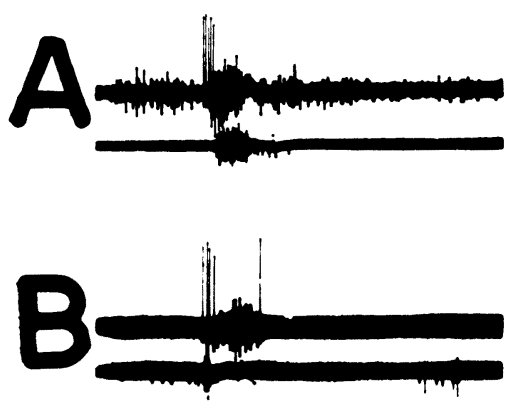
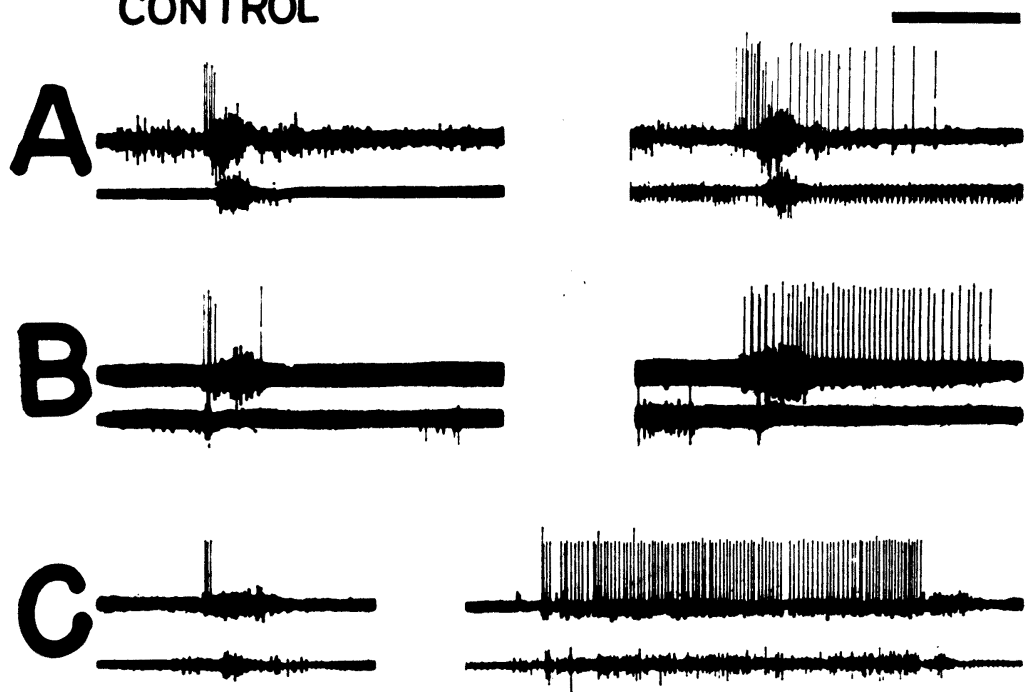

FIG. 1. Alterations in the activity patterns of the hypoglossal nerve fibers during swallowing in the kitten under different conditions (right column). A: light asphyxia, B: squirting a large amount of water, C: mechanical touch to the pharyngeal surface. Control responses were obtained by squirting a small amount of water into the oropharynx of the animal while it was breathing quietly. Upper trace: motor activity of the hypoglossal nerve fiber. Lower trace: activity of the geniohyoid muscle. Time bar: $1 \mathrm{sec}$. 
hypoglossal nerve fiber during swallowing in the adult animal is very stable, and the alteration under the different conditions described above could scarcely be produced.

The difference in stability of the swallowing discharge between kittens and adults can be recognized by comparing the mean mode of discharge determined statistically from a number of samples for the swallowing discharges repeatedly induced in the respective hypoglossal nerve fiber. As shown in FIG. 2, the swallowing discharges in the adult (A, B, C and D) revealed a duration ranging from 0.15 to $0.3 \mathrm{sec}$ and a slight deviation in discharge frequency of impulses from the mean. In the kitten ( $E, F, G$ and $H$ ), however, they revealed a much longer duration and wider range of deviation in the discharge frequency of impulses. These facts indicate the stability difference in the discharge pattern between kittens and adult cats. The stability is assumed to be accomplished during the course of pre-and postnatal development of kittens roughly before they become 3 month old.

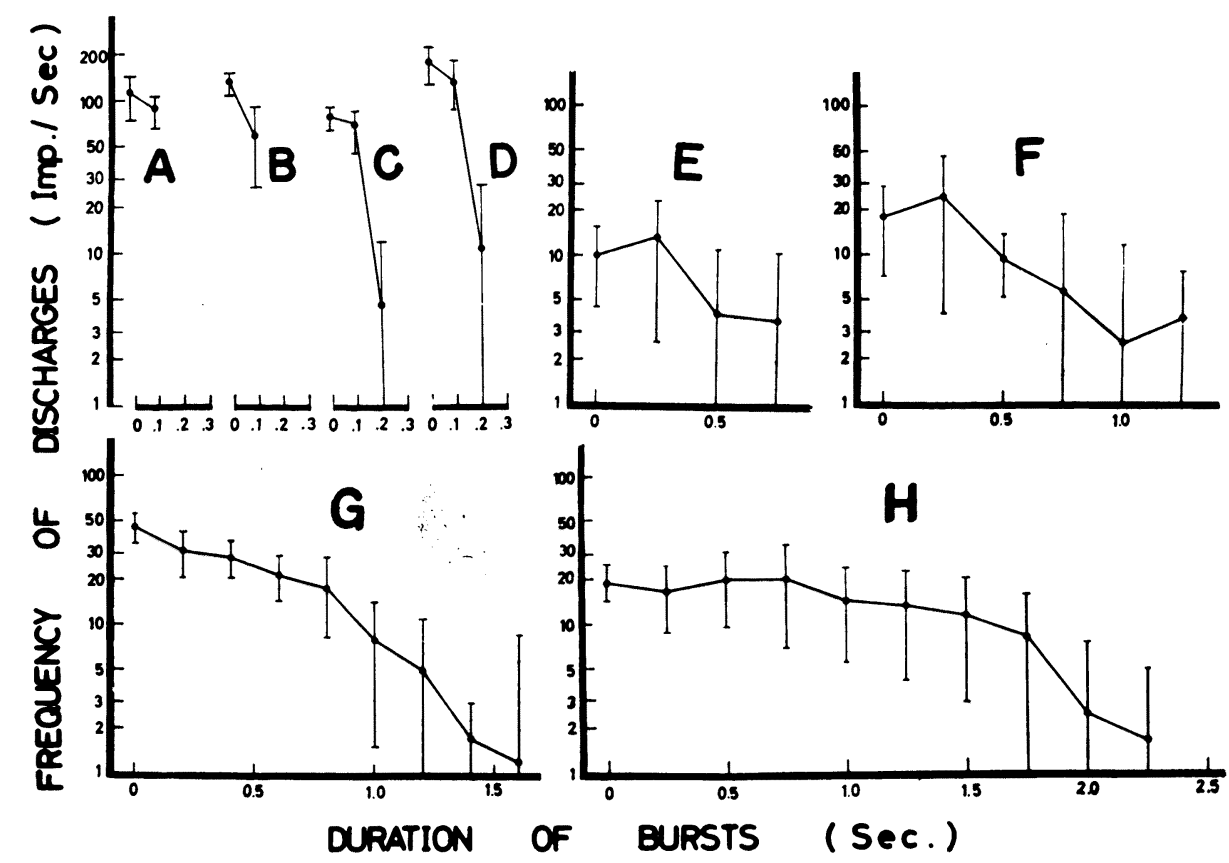

Fig. 2. Comparison in discharge frequency and duration of the hypoglossal nerve fibers during burst activity of swallowing between adult cats (A, B, C and $D$ ) and kittens (E, F, G and $H$ ). Swallowing was induced by squirting a small amount of water into the oropharynx. The frequency (ordinate) calculated from the spikes discharged during each $0.1,0.2$ or $0.25 \mathrm{sec}$. was plotted against the time (abscissa) after the onset of the burst activities. The height of each vertical bar represents twice the standard deviation of the mean frequency of different bursts for the identical unit. 
2. Effects of motoparalysis on the "swallowing discharges" of the hypoglossal nerve fibers. As previously reported ${ }^{12)}$ the burst discharges of hypoglossal motor fibers in the adult cat during swallowing revealed no substantial modification in their pattern after motoparalysis was induced by intravenous administration of Flaxedil. The procedure, on the contrary, gave rise to a profound alteration in the discharge of the kitten hypoglossal nerve fiber. Namely, after motoparalysis the squirting of tap water into the oropharyngeal cavity did not produce any spike in the hypoglossal nerve fiber or produced a very few spikes with a complete abolishment of the act of swallowing. The effect, however, gradually became enlightened with the lapse of time until finally restored to the control appearance. This can clearly be seen from the serial records in FIG. 3.

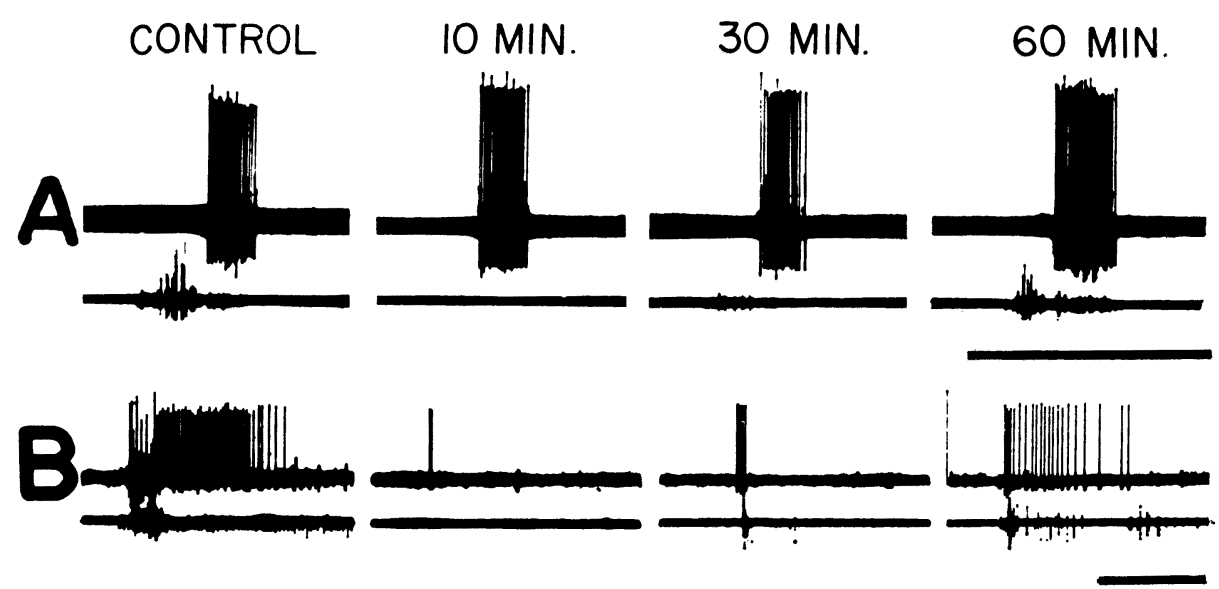

FIG. 3. Contrasting examples of the effects of motoparalysis on the "swallowing discharge" in the cat (A) and kitten (B). Each burst of swallowing was elicited by squirting water into the oropharynx. Patterns of the discharge of the hypoglossal nerve fiber (upper traces) are unmodified in $\mathrm{A}$, however, diminuted prominently in B both in the number of spikes and in the discharge duration after motoparalysis. Responses of the geniohyoid muscle (lower traces) associated with swallowing are similarly depressed, thereafter recovering gradually with the lapse of time (indicated at the top) after administration of gallamine triethiodide. Time bar : 1 sec.

The effect of motoparalysis on the hypoglossal nerve fiber activity was different from that described above when the activity was induced by electrical tetanic stimuli to the central stump of the superior laryngeal nerve. In the control conditions, the stimuli produced repetitive bursts of impulses in the hypoglossal nerve fiber associated with the act of swallowing. The frequencies of the repetitive bursts were approximately 12 to 18 per minute in the adult (cfr. (1): 20 swallows/min. for dogs, 8 swallows/min. for rabbits) and 55 to 60 per minute in the kitten (FIG. $4 \mathrm{~A}$ and B, top records). During 
the interval between bursts, no or very slight activity was produced. After motoparalysis the repetitive bursts of swallowing can be constantly produced in the adult cat with a slight decrease in frequency to 10 per minute (FIG. $4 \mathrm{~A}$, second record) while the activity in the geniohyoid muscle failed to occur until a gradual recovery was attained as time elapsed (FIG. 4A, third and bottom records). In the kitten, on the other hand, motoparalysis elicited a prominent alteration in the activity pattern of the hypoglossal fiber during the superior laryngeal nerve activation by electrical stimuli, namely the motor discharge lost its grouping and periodic nature and tended to reveal a continuous train of impulses, especially during initial period of the electrical stimulation. The result well coincides with the one in a 3-day-old kitten reported by DоTY, i.e. the repetitive swallowing could not be obtained and there was a tendency for a single swallow in response to various periods of superior laryngeal nerve stimulation ${ }^{2)}$. The active period thereafter was followed by a complete silence. Throughout the period of electrical stimulation, no sign of activity in the geniohyoid muscle could be seen. The alteration of response both in the hypoglossal nerve fiber and in the geniohyoid muscle, however, was restored gradually after a further elapsing of time following the injection of the paralysing agent (FIG. 4B, bottom record). The type of activity modification caused by motoparalysis in the hypoglossal nerve fiber of kittens, either during swallowing induced by squirting water into the oropharynx or during afferent excitation of the superior laryngeal nerve by

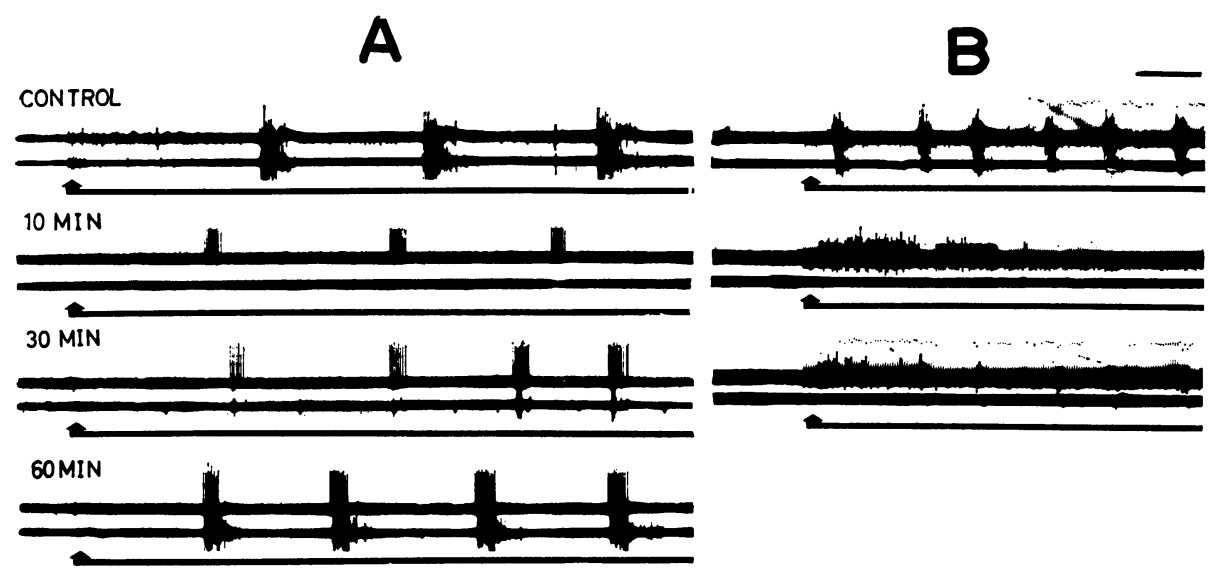

FIG. 4. Effects of motoparalysis on the responses of the hypoglossal nerve fiber to tetanic stimulation of the superior laryngeal nerve in the adult cat (A) and kitten (B). No essential change in $A$, while abolition of the repetitive nature in $B$ by motoparalysis can be seen (note upper trace in the second record from the top which was obtained 10 minutes after the Flaxedil administration). Activities of the geniohyoid muscle (lower traces) in A and B are similarly inhibited by motoparalysis. Lines with arrows pointing upward indicate the timing and duration of the tetanic stimulation. Time bar: $1 \mathrm{sec}$. 
repetitive stimuli, gradually attained to that obtained in the adult cat. The critical stage for the completion of the development was at around 3 month postnatally.

3. Effects of swallowing on motor activity of the phrenic nerve fibers. As shown in FIG. 5A, the phrenic nerve fibers revealed the respiratory activity and the majority of the fibers examined responded, together with the initiation of a swallowing, to the squirt of water into the oropharyngeal cavity by displaying a burst discharge of 3 or 4 impulses for $0.1 \mathrm{sec}$. The response was always followed by a period of silence of 1.0 to $1.5 \mathrm{sec}$. before the next normal respiratory volley began. The duration of this silent period was independent to the phase of respiration during which the burst response was elicited. A normal respiratory volley following the burst response did not reappear at the time it should have, as predicted by the duration of the respiratory cycle, but only began after a delay that was constant for individual fibers. The respiratory volley thus reappeared after the burst response revealed an enhancement.

The phrenic fibers that responded with the burst discharge of impulses by the squirt of water also produced the repetition of such bursts when tetanic electrical stimuli was applied orthodromically to the superior laryngeal nerve. Throughout the whole period of stimulation, the spontaneous respiratory volley was completely suppressed (FIG. 5B). In a few cases, however, the respiratory volley was gradually released from the suppression during the later stage of stimulation. Thus, the repetitive swallowing bursts and the respiratory volleys became intermingled (FIG. 5C). Very few of the

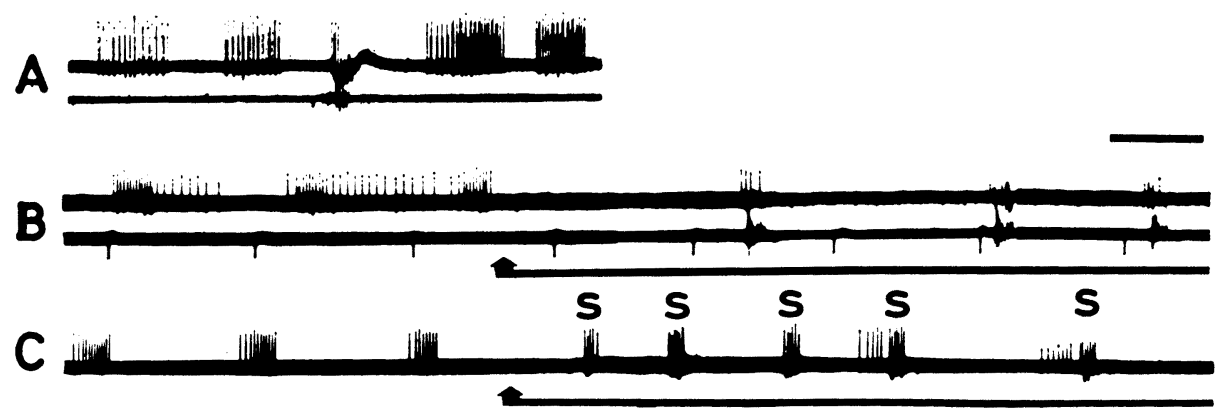

FIG. 5. Changes in the activity patterns of phrenic nerve fibers caused by swallowing (A) and by tetanic stimulation to the superior laryngeal nerve (B and C) in the kitten. A : "swallowing burst" elicited by squirting a small amount of water into the oropharynx. B: conversion of the rhythmical respiratory activity to the repetitive bursts associated with swallowing. $\mathrm{C}$ : intermingled activity of the respiratory and the repetitive swallowing bursts (s). Lines with arrow pinting upward indicate the timing and duration of stimulation (30 pulses/sec.) Time bar: $1 \mathrm{sec}$. 
phrenic fibers ceased the respiratory activity when a swallow was produced during the firing phase. The time relation between the onset of quiscence by a swallow and the reappearance of normal respiratory volley in this type of fiber revealed similarity to that obtained in the fiber that responded with a burst activity to swallowing.

4. The reflex response of the phrenic and hypoglossal nerve fibers to single shock stimuli to the superior laryngeal nerve. In kittens, the responses of the nerve fibers of the phrenic and the hypoglossal nerves to single shock stimuli to the superior laryngeal nerve were recorded to obtain the knowledge on the development of the neuronal connections along the reflex pathways. As shown in FIG. 6B, the response of the phrenic nerve fiber usually consisted of multiple spikes except when the stimulus intensity was just above the threshold. The latency of the first spike of the response ranged from 28 to 35 msec., longer than that in the hypoglossal motor fiber during the same procedure (FIG. 6A), i.e. ranging from 17 to $20 \mathrm{msec}$. (cfr. 12 to $15 \mathrm{msec}$. in adult cats $^{12}$ ) and the long latency of the reflex response in the kitten is due to slow conduction velocity of the peripheral nerve fibers and their intramedullary extensions ${ }^{9,13}$. Even though the time difference due to the length difference of the two reflex pathways is considered, the central reflex time for the former is longer than that for the latter. This may imply that the superior laryngeal-phrenicus reflex is functioning through a more complex neuronal organization than the one analogous to the inter-segmental reflex in the spinal cord.

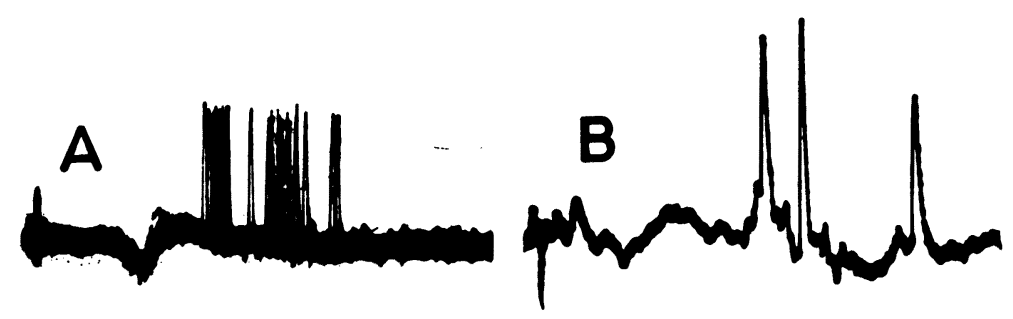

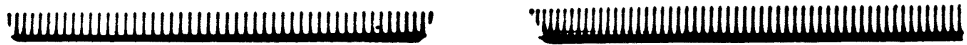

FIG. 6. Responses of the motor fiber of the hypoglossal (A) and phrenic (B) nerves to single electrical shocks applied to the superior laryngeal nerve in the kitten. In A. 10 traces were superimposed. Note the difference in response latency. Time marks: msec.

5. Modification of the activity pattern of the hypoglossal motor fibers during asphyxia or afferent stimulation to the superior laryngeal nerve. The hypoglossal motor unit, shown by the record at the top-left in FIG. 7, revealed no spontaneous activity when first encountered and responded typically with the 
"swallowing burst" when squirting water into the oropharyngeal cavity. When asphyxia was produced, a new unit, the one which did not reveal any response to the squirt of water previously, gradually became active yielding the respiratory rhythm of discharge. During the advanced stages of asphyxia, the "swallowing unit" was recruited into the respiratory activity (FIG. 7 middle record). In this case, however, the two units were active reciprocally and it was later identified that the unit of large spike was inspiratory and the other was expiratory. With a further increase of asphyxia, the respiratory reciprocity was lost and the two units showed a simultaneous and continuous train of impulses (FIG. 7 , bottom record). At the terminal stage of
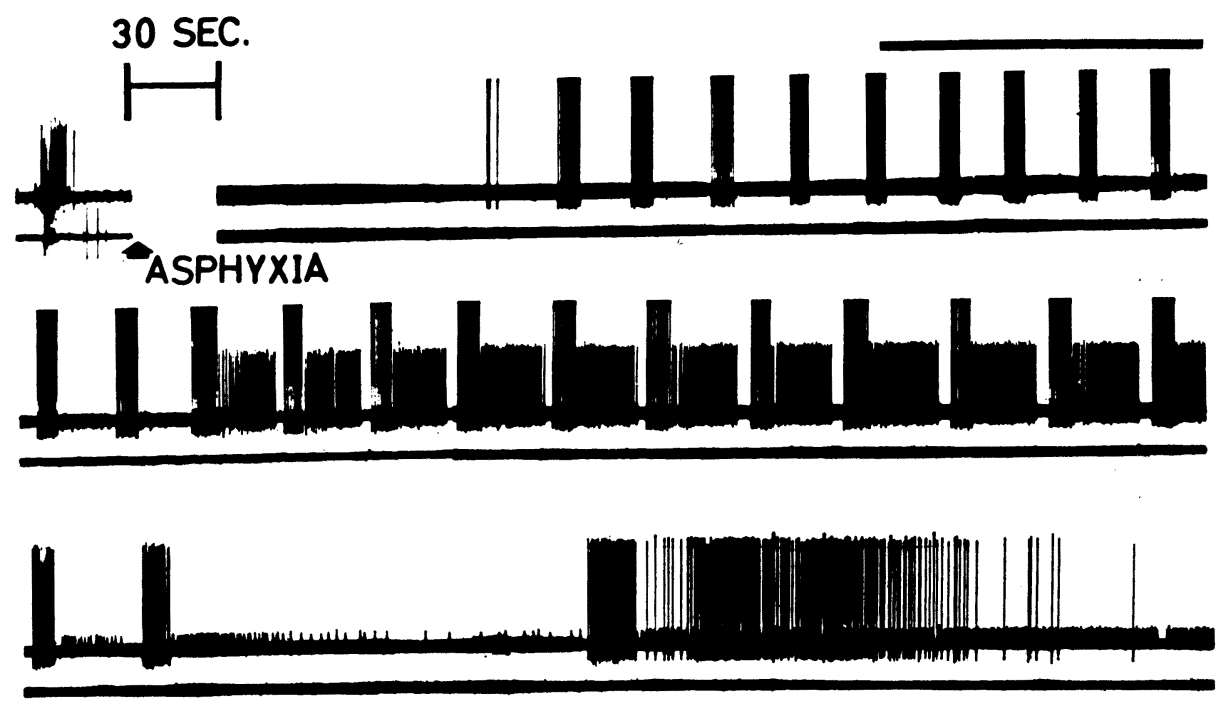

FIG. 7. Effects of asphyxia on the activity of the hypoglossal nerve fiber in the 4 week old kitten. The activity of the fiber during swallowing caused by squirting water into the oropharynx is shown at the top-left corner. A continuous record from left to right and from top to bottom taken during asphyxia (period beginning at the moment indicated by the arrow pointing upward till the end). Record at the bottom was obtained under a decreased amplification. Time bar: $10 \mathrm{sec}$.

asphyxia, the continuous activity changed into another type of rhythmical activity, the gasping. The conversion of the activity pattern described above could be elicited in the majority of hypoglossal motor fibers of the kittens examined. The minority revealed, under light aspyxia, the activities of random impulses or of irregular bursts of impulses. In the adult cat, on the other hand, as reported previously ${ }^{12}$, asphyxia produced in some hypoglossal motor fibers an activity of spontaneous bursts of swallowing without locking to the rhythm of respiration.

A few hypoglossal motor fibers yielded the respiratory rhythm of impulses 
even under eupneic condition. When the superior laryngeal nerve was stimulated by repetitive pulses, such type of unit responded simultaneously with the "swallowing unit" by generating only 1 to 3 impulses in one swallowing. This resembled to the behaviour of the respiratory motor fibers in the intercostal $^{10)}$ or phrenic nerves.

\section{DISCUSSION}

The present results indicate that the integrative action of nerve cells in the brain stem is not well developed functioning to accomplish the reflex swallowing in the early stage of postnatal life of kittens. It is of considerable interest that the reflex took muck longer period for the development than the myelinated fibers in cat sciatic nerves ${ }^{9)}$ or the mono- or polysynaptic reflexes in the spinal $\operatorname{cord}^{6,8,13)}$. This may indicate a more complex neuronal arrangement for the reflex deglutition in the brain stem. Contrary to what was shown in the adult cats, the dependance and compliance of the discharge of hypoglossal motor impulses upon the pattern of sensory impulses from the oropharyngeal structures (FIG. 3 and 4) suggest 1) the immatured ability to build up autonomically the pattern of "swallowing discharge" in the brain stem nerve cells concerned and 2) the importance of the feedback of the sensory impulses from the oropharyngeal structures to complete the swallowing act in the kitten.

The long latency of the reflex response of the phrenic motor fiber to the superior laryngeal nerve stimulation with single shocks, as compared to that of the reflex response of the hypoglossal nerve fiber, is due to 1) the longer pathway and/or 2) the involvement of more synapses in the superior laryngealphrenic nerve reflex than in the other. In the present case, however, the latter factor seems to be essential since the difference of the latencies between the two reflexes was far greater than the time difference calculated from their conduction distance. It would be most probable to postulate therefore that the sensory impulses in the superior laryngeal nerve fiber impinge first into the cells of solitary nucleus ${ }^{12)}$ then reach at the phrenic motoneurons with an intervention of neuronal organization in the medulla subserving respiration and/or swallowing.

The phrenic nerve fibers of kittens responded to the squirt of water into the oropharyngeal cavity by displaying a burst discharge of 3 to 4 impulses for $0.1 \mathrm{sec}$. As previously reported in adult cats, however, the burst comprised of impulses of 3 up to 24 for $0.13 \mathrm{sec}$. in the group of medullary inspiratory neurons, less than 2 impulses in the inspiratory motoneurons of the thoracic spinal cord and 7 to 50 in the motoneurons of the hypoglossus and the ambiguous nuclei respectively ${ }^{10,11,12}$. Thus, it appears that the burst response of phrenic motoneurons associated with swallowing was of an inter- 
mediate degree between that obtained in the inspiratory neuron group in the brain stem and that in the spinal cord at the intercostal level. This assumption, however, requires further evidences before it becomes valid.

A widely dispersed occurrence of the burst activity in the fibers of the phrenic and accesssory nerves in additions to the motor nerve fibers innervating the proper "swallowing muscles" in the kitten is not surprising when one considers that swallowing is phylogenetically an early form of respiration? and that the two functions are so locked in mammals that one cannot occur together with the other. It has been postulated ${ }^{12)}$ that the respiratory and the swallowing networks of cells in the brain-stem each has a "hard core" of units constantly locked to their own specific functional modality while others can be recruited when necessary for the other modality. This is likely to be more substantial than the idea that the "respiratory center" interrelates with the "swallowing center" by irradiation of excitation or inhibition from one center to the other mutually ${ }^{4,5}$. The degree of dispersion for the swallow response and the number of impulses in such a burst are more pronounced in the kitten than in the adult cat. From these and the data presented in section 3 and 5 of Results, the interrelation between central regulatory mechanisms for swallowing and respiration seems to be more close in the kitten and the differentiation between the two functions progresses postnatally.

\section{SUMMARY}

1. The nature and course of postnatal development of reflex deglutition were investigated by means of the single fiber recordings from the motor fibers of hypoglossal, accessory and phrenic nerves in unanesthetized, midcollicularly decerebrate cats and kittens of various postnatal periods.

2. The motor fibers of kitten hypoglossal nerve showed a great deal of variety of patterns of discharge during swallowing depending on the differences of the nerve fiber, the squirt of water into the oropharynx, the type of mechanical stimulation and the ventilatory state of the animal. The burst discharge was also produced in the nerve fiber innervating the muscles having no direct participation in the act of swallowing.

3. Gallamine-induced motoparalysis modified profoundly the pattern of the motor nerve fiber discharges both during swallowing and during electrical stimulation of the superior laryngeal nerve in the kitten.

4. The hypoglossal motor fibers which revealed the "swallowing discharge" in normal conditions produced the respiratory volleys when the animal was asphyxiated, whereas the phrenic motor fibers discharging rhythmically in phase with respiration produced the "swallowing discharge" (a complete silence in a few fibers) when a small amount of water was squirted into the oropharynx or the superior laryngeal nerve was stimulated electrically by 
repetitive pulses.

5. The response features of the motor nerve fibers of kitten hypoglossal nerve during swallowing gradually approached to those of the adult cats with the lapse of time in approximately 3 months postnatally.

A part of the expenses for the study was defrayed by the grant from the Ministry of Education of Japan.

\section{REFERENCES}

1) Bosma, J. Deglutition: pharyngeal stages. Physiol. Rev. $37: 275-300,1957$.

2) Dоту, R. Influence of stimulus pattern on reflex deglutition. Amer. J. Physiol. 166 : 142-158, 1951.

3) Doty, R. And J. Bosma. An electromyographical analysis of reflex deglutition. J. Neurophysiol. $19:$ 44-60, 1956.

4) Hukuhara, T. And H. OKada. Effects of deglutition upon the spike discharges of neurons in the respiratory center. Jap. J. Physiol. 6: 162-166, 1956.

5) MARCKWARD, M. Über die ausbreitung der Erregung und Hemmung von Schluckzentrum auf das Atemzentrum. Z. Biol. 25, 1-54, 1889.

6) NAKA, K. Electrophysiology of the fetal spinal cord. J. Gen. Physiol. 47 : 1003-1038, 1964.

7) Negus, V.E. The comparative anatomy and physiology of the larynx. London, Hafner, 1962.

8) Skoglund, S. On the postnatal development of postural mechanisms as revealed by electromyography and myography in decerebrate kittens. Acta Physiol. scand. 49: 299-317, 1960.

9) Skoglund, S. The spinal transmission of proprioceptive reflexes and the postnatal development of conduction velocity in different hindlimb nerves in the kitten. Acta physiol. scand. 49 : 318-329, 1960.

10) Sumi, T. The activity of brain-stem respiratory neurons and spinal respiratory motoneurons during swallowing. J. Neurophysiol. $26: 466-477,1963$.

11) Sumi, T. Spinal respiratory neurons and their reaction to stimulation of intercostal nerves. Pfiügers Arch. Ges. Physiol. 278 : 172-180, 1963.

12) Sumi, T. Neuronal mechanisms in swallowing. Pflïgers Arch. Ges. Physiol. 278 : 467-477, 1963.

13) Wilson, V. J. Reflex transmission in the kitten, J. Neurophysiol. 25 : 263-276, 1962. 